\title{
Following model for ships in navigation tunnels
}

\author{
Gan Weidong ${ }^{1}$, Ma Dianguang ${ }^{1}$, Li Yanwei ${ }^{1, a}$ and Deng Jian ${ }^{2}$ \\ ${ }^{1}$ Tianjin Research Institute of Water Transport Engineering, Ministry of Communications, 300456 Tianjin, China \\ ${ }^{2}$ School of Navigation, Wuhan University of Technology, 430063 Wuhan, China
}

\begin{abstract}
Determination of the appropriate following distance between ships is essential for their safe passage through navigation tunnels. In this study, it uses the following theory of traffic flow to establish a model that enables the calculation of the following distance between ships in a navigation tunnel. Based on the proposed model, the study also introduces an approach to calculate and control the following distance at different risk levels in order to control the passage intervals within a navigation tunnel. In addition, the study tests the model using data for the second line navigation tunnel of the Silin Junction in the Wujiang River, China and compare the results with existing distance control parameters for ships in the Goupitan first line navigation tunnel in the Wujiang River. The proposed model can effectively reduce the risk of ship navigation in tunnels by providing reference information for safe ship navigation, thus allowing operational and management staff to ensure adherence to safety procedures within the navigation tunnel.
\end{abstract}

\section{Introduction}

To accelerate the economic growth of a country, its transportation infrastructure needs to be overhauled, including that for inland water transportation. In particular, initiatives towards improving inland water transportation in China have involved addressing existing shortcomings by focusing on principal channels, such as the Yangtze River; building a high-quality channel network connecting the main branches of rivers to the sea; promoting standardization and proper cleaning of ships; intensifying the construction and modernization of ports; achieving organic connection between land and sea; and building a network of waterways connecting the entire basin.

At present, efforts are being undertaken to widen several mountainous rivers in Southwest China so that they become more navigable. The abundance of dams and extensive canyon topography in these regions has led to the widespread use of navigation tunnels to allow vessels to pass through mountains and dams. However, navigation tunnels often have low visibility, small section coefficients, and limited surplus scalability. Consequently, they are generally straight and used for one-way navigation, and ships can only navigate them sequentially without the possibility of overtaking. To ensure safety and prevent accidents, a safe distance between ships must be maintained and strictly controlled.

Research into the safe distance between ships typically employs methods such as ship domain theory [1-3], following theory [4] and statistical analysis theory [5-8]. Following theory was developed to study the flow of ground vehicle traffic in a single lane and focuses on the reaction of the trailing car when the speed of the leading car changes. However, its use has widened and ship following theory is now commonly employed to analyze and model factors such as the braking distance of ships and the reaction time of navigators [9]. According to the existing research results, the following theory can only be applied in the field of ships under specific conditions. thus, specific conditions need to be modified in these models to ensure that they are applicable to ship navigation, including the abovementioned one-way single-ship navigation.

Existing research that applies following theory to ships primarily focuses on ground-level channels with good visibility.which are on the ground and broad vision. For instance, He et al. [10] analyzed the relationship between ship braking distance and its length and speed to derive an empirical formula for this distance. In addition, they considered the influence of crew experience, established a model for the following distance of ships based on their analysis, and determined the distance model parameters based on practical examples. Meanwhile, Ming et al. [11] established a model for longitudinal safety distance of super-large ships navigating through a deep-water route. Li and Sun [12] adapted the following model for land traffic flow to a following model for navigation safety of ships traveling the Arctic route. In contrast, Cheng et al. [13] used following theory and information on ships' inertial and reverse stopping distances to develop a model for safe following distances in inland waterways under low visibility. The results of this body of work imply that

\footnotetext{
${ }^{\mathrm{a}}$ Corresponding author: 542774678 @qq.com
} 
following theory can only be applied to ships under the specific conditions of one-way, single-ship navigation

In this study, it considers the special environment of navigation tunnels and the constraints of ships navigating within them. Using the operation theory of ships and following theory of traffic flow, it proposes a following distance model for ships passing through navigation tunnels. In addition, it proposes an approach for the management and control of ships in these navigation tunnels, thus providing a solution for their safe and efficient operation.

\section{Following model for ships}

\subsection{Ship maneuverability}

\subsubsection{Ship parking distance}

The parking distance of a ship refers to the distance travelled by the center of gravity of the ship from the moment that the engines stop until the ship stops moving through the water. The time taken to complete the maneuver is known as the parking time. Parking distance $\left(S_{P}\right)$ and parking time $\left(t_{s}\right)$ can be expressed as [14-15]:

$$
S_{P}=\frac{\rho_{w} \cdot \nabla \cdot\left(1+k_{1}\right)}{k} \cdot \ln \frac{v_{0}}{v}
$$

and

$$
t_{s}=C \log _{2} \frac{v_{0}}{v}
$$

where $\rho_{w}$ is the density of water, $\nabla$ is the displaced volume, $k_{1}$ is the longitudinal drag coefficient, $k$ is the resistance coefficient, $v_{0}$ is the initial speed, $v$ is the speed of ship at a certain moment before it stops, and $C$ is the time constant of halving the ship's speed.

\subsubsection{Ship reversing distance}

Ship braking is usually implemented as a full-speed reverse. The reversing distance of a ship describes the distance that the ship travels forwards from the onset of the application of reverse thrust until the vessel stops moving through the water[16]. The reversing distance is also known as the emergency stopping distance or the shortest stopping distance.

A ship's reversing distance $\left(S_{R}\right)$ can be written as:

$$
S_{R}=L_{s} \cdot[\alpha \cdot \ln (1+\beta)+\delta] \text {, }
$$

where $L_{s}$ is the length of ship, $\alpha$ is the ratio of ship's mass to its drag, $\beta$ is the ratio of the ship's drag before the ship's reverses to the reverse thrust, and $\delta$ is the product of the time when the ship generates reverse thrust and its initial speed when it starts reversing.

\subsection{Following Theory Application}

The following model for vehicles uses a dynamic method to determine the following state when vehicles cannot pass in one-way lanes. Three basic characteristics of vehicles in the following state constitute the model principles, namely conditionality, delay, and transitivity [17]. In navigation tunnels, ships travel sequentially and cannot overtake those in front; hence, a braking ship affects the trailing ships similarly to a braking car in the one-way lane model for road traffic. Therefore, the proposed safe distance model for ships is based on the principles of road following theory.

If a ship sailing within a navigation tunnels stops because of mechanical failure or emergency (Stage 0), the trailing ships must be able to stop safely, imposing the necessity of a safety margin (distance) between adjacent ships to avoid collisions. The low-visibility environment within navigation tunnels hampers the ability of the navigator to suddenly slow or stop the trailing ship because they often rely on visual input to execute timely maneuvers. Thus, the navigator of the leading ship must inform those of the trailing ships via VHF broadcast of any unexpected situation to allow time for the trailing ships to perform their braking procedure, which typically involves four stages. In Stage 1, a notification is sent by the navigator of the leading ship to the other ships. In Stage 2, a braking reaction is undertaken by the navigator on the trailing ships. The braking operation is triggered by the trailing ship in Stage 3. Finally, in Stage 4, continuous braking of the ship occurs. These stages are illustrated in Figure 1.

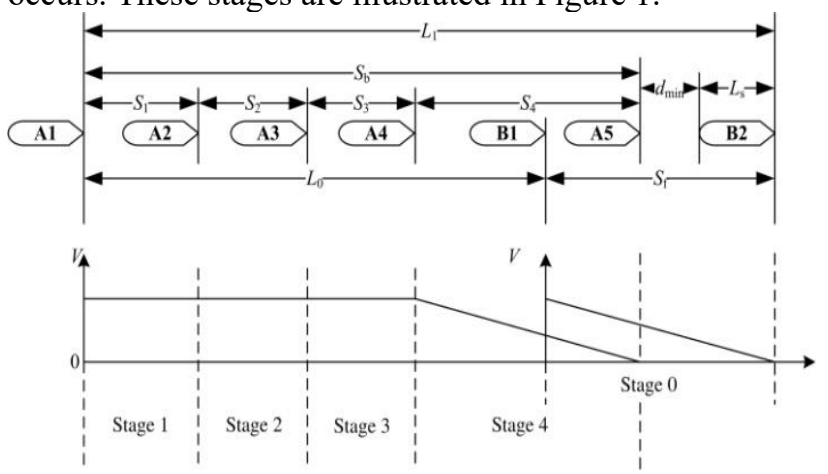

Figure 1. Stages of braking behavior for ships traveling in a one-way navigation tunnel.

The time at which the leading ship begins braking is taken as the initial time of the braking sequence and the time at which braking ceases is the final time. Throughout braking, the trailing ship within the tunnel occupies the five positions (A1 to A5) illustrated in Figure 1, while the leading ship occupies two positions (B1 and B2). The distance (in meters) between stopped 
position (B2) of the leading ship and the initial position (A1) of the trailing ship is given by:

$$
L_{1}=L_{0}+S_{f}
$$

where $L_{0}$ is the distance between the bows of the two ships when the leading ship begins braking and $S_{f}$ is the distance covered by the leading ship with its engine off, or that covered before it stops completely.

During the period between initial braking by the leading ship and its final stopping, the trailing ship covers the distance $S_{b}$ :

$$
\begin{gathered}
S_{b}=S_{1}+S_{2}+S_{3}+S_{4}, \\
S_{1}=v t_{1}, \\
S_{2}=v t_{2},
\end{gathered}
$$

and

$$
S_{3}=v t_{3},
$$

where $S_{1}$ is the distance that the trailing ship travels during the radio broadcast from the leading ship (and $t_{1}$ is the duration of the radio broadcast), $S_{2}$ is the distance that the trailing ship travels while its navigator is assessing the motion of the leading ship after acknowledging the broadcast (and $t_{2}$ is the time taken to achieve this), $S_{3}$ is the distance that the trailing ship travels in time $t_{3}$ while its navigator is engaging the brake, $S_{4}$ is the braking distance of the trailing ship, $v$ is the sailing speed (in meters per second) of the trailing ship.

After the ships have completely stopped, the safety distance between them should remain greater than the minimum stipulated distance, $d_{\min }$ :

$$
L_{1}-S_{b}-L_{s} \geq d_{\text {min }},
$$

where $L_{s}$ is the length of the leading ship.

By substituting Equations (4)-(8) into (9), the following distance relationship between ships in the navigation tunnel is obtained:

$L_{0} \geq d_{\min }+L_{s}+v\left(t_{1}+t_{2}+t_{3}\right)+S_{4}-S_{f}$,

where $L_{0}$ is the following distance. Assuming the same braking performance between the leading and trailing ships, the following distances at three different states can be obtained:

1) If the leading ship is travelling with the engine stopped, $L_{0}$ is a dangerous following distance:

$$
L_{\min }=d_{\min }+L_{s}+v\left(t_{1}+t_{2}+t_{3}\right)+S_{4}-S_{f} .
$$

2) If the ships are reverse braking, $L_{0}$ is a normal following distance:

$$
L_{\min }=d_{\min }+L_{s}+v\left(t_{1}+t_{2}+t_{3}\right) \text {. }
$$

3) If the ships in front hits the bottom, sustains damage, and suddenly stops, $L_{0}$ is a safe following distance:

$$
L_{\min }=d_{\min }+L_{s}+v\left(t_{1}+t_{2}+t_{3}\right)+S_{4}
$$

\section{Distance control interval and following model for ships in navigation tunnels}

Given the so-called black-hole effect at the entrance of the navigation tunnel and the white-hole effect at its exit [18-19], ships outside the entrance might not see those within the navigation tunnel, and those close to the exit might not see the ships outside the tunnel. Therefore, the following distance for the ships should be the same either within or outside the navigation tunnel, corresponding to a complete following distance, $L_{0}$, as shown in Figure 2.

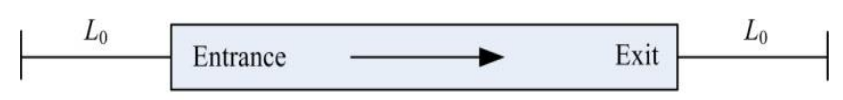

Figure 2. Navigation tunnel and following distance of ships outside the tunnel.

Given the operating characteristics of the navigation tunnel and the reactions of ship navigators, it is difficult for ships in front of or behind a disabled ship to know its position. Therefore, other ships do not know whether braking measures should be initiated, even if timely information of the situation is conveyed via VHF broadcast. Therefore, it considers that all ships should be numbered and controlled when sailing within navigation tunnels by adopting the following procedure:

1) Ship numbering. Every day, at 00:00, the first upstream ship is numbered as upper 1 when it enters the downstream navigation lock or ship lifter, the second ship is numbered as upper 2, and so on until 00:00 of the next day.

2) Distance control. The distance between adjacent ships should be maintained as required.

3) Dynamic broadcast. If the speed of a ship changes, the adjacent ships are informed of this via VHF broadcast. The content of the broadcast message should include the ship number and its dynamic situation.

4) Emergency response. Each ship should determine whether it is in front of or behind a broadcasting ship by referring to the number in the broadcast message and its own number. If it is ahead of the broadcasting ship, it should continue sailing, but it should stop immediately if behind.

\section{Model application}

\subsection{Navigation tunnel}

The second line navigation structure of the Silin hydropower station in the Wujiang River is composed of an upstream approaching channel, reservoir lock, open 
channel 1, flood control gate, navigation tunnel, open channel 2, vertical ship lifter, and downstream approaching channel, among other structures, as shown in Figure 3 . The length of the navigation tunnel is $2573 \mathrm{~m}$, its cross-section is $16 \mathrm{~m} \times 20 \mathrm{~m}$ (width $\times$ height), and the water depth within the tunnel is $5.5 \mathrm{~m}$. It used the dualtunnel one-way navigation scheme, in which ship overtaking is forbidden within the tunnel, and the ship speed is limited to $2.5 \mathrm{~m} / \mathrm{s}$. The representative ship type is a 1000-ton container (the typical ship traveling along the Wujiang River) with dimensions of $55.8 \mathrm{~m} \times 10.8 \mathrm{~m}$ $\times 2.5 \mathrm{~m}$ (length $\times$ width $\times$ draft $)$.

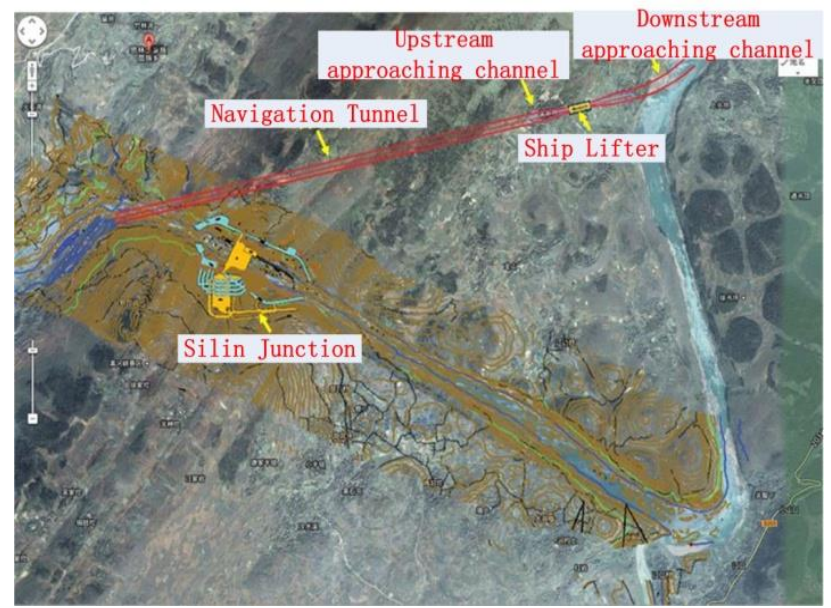

Figure 3. Layout of the second navigation line of the Silin Dam in the Wujiang River.

\subsection{Parameter settings}

The primary factor affecting distance $S_{1}$ is the broadcast period. Considering the amount of information in the broadcast message, the time for the navigator in the leading ship to finish broadcasting is $10-20 \mathrm{~s}$.

Distances $S_{2}$ and $S_{3}$ depend primarily on reaction time (an important parameter characterizing the physiological and psychological status of navigators), including consciousness time (2-3 s) and action time (35 s) [11].

$S_{4}$ and $S_{f}$ reflect the inertial stopping distance or reverse stopping distance of the ship, depending on the situation. They can be estimated using empirical formulas and verified by operation simulations of large ships. The inertial stopping distance of a 1000-ton container ship is $46 \mathrm{~m}$ at $2.5 \mathrm{~m} / \mathrm{s}$, while the reverse stopping distance is 30 $\mathrm{m}$. When two ships are stationary in the water and there is no collision, $d_{\min }$ is zero.

\subsection{Calculation of following distance}

It assumes that the maneuverability of the ships in the navigation tunnel is the same. Accordingly, the following distances for ships are listed in Table 1.

Table 1. Following distances for ships in the Silin Dam Navigation Tunnel on the Wujiang River.

Level

Distance (m)

\begin{tabular}{ll}
\hline Dangerous following distance & 109.8 \\
Normal following distance & 125.8 \\
Safe following distance & 155.8 \\
\hline
\end{tabular}

\subsection{Control of following distance}

Considering safety as the most important factor in navigation, the safe following distance should be maintained as the operational following distance. Therefore, the control interval length for the shipfollowing distance within the second line navigation tunnel in the Silin Junction of the Wujiang River is $2884.6 \mathrm{~m}$.

According to trial operations involving the Goupitan first line navigation tunnel, ships should maintain a distance of $100 \mathrm{~m}$, which is a value consistent with the one obtained using our proposed model. In practice, however, the following distance of ships within navigation tunnels is usually determined based on the individual experience of navigators, which could potentially lead to safety risk.

\section{Conclusions}

In this study, it established a model for the determination of ship following distances within navigation tunnels using the following theory for traffic flow. The proposed model enables calculation of ship following distances at different risk-levels within a navigation tunnel. In addition, it obtained credible results when applied the proposed following distance model for the second line navigation tunnel in the Silin Junction in the Wujiang River. However, a limitation of our study is that our proposed model assumes that the maneuverability of the leading and trailing ships is similar; whereas, differences between ships affect navigation. Thus, the effect of differences in ship handling on safe following distances needs to be explored further.

\section{References}

1. FUJII Y, TANAKA K. J, Nav. 24, 543-552 (1971)

2. GOODWIN E.M, J. Nav. 28, 328-344 (1975)

3. DAVIS P.V,M.J. Dove, J. Nav. 33, 215-222 (1980)

4. J. Zhu, W. Zhang, Traffic Transp. Eng. 9, 83-87 (2009)

5. H.Q. Hou, Y.C. Li, X.M. Chu. J, Dalian Marit. Univ. 39, 21-24 (2013)

6. J. Yu, W. Zhang, Traffic Transp. Eng. 6, 88-93 (2006)

7. X.M. Chu, Y.C. Li,Y.H. Yu, Transp Syst. Eng. Infor. Tech. 14, 213-219 (2014)

8. L.Y. Chen, J.M. Mou, J.F. Dai, et al., Navig. China, 36, 76-81 (2013)

9. Z. Wu, X.J. Wang, Guizhou Univ. 31, 118-122 (2014)

10. L.D. He, Y. Jiang, Z.J. Yin, B. Zhou, H. Tang, J, Traffic Transp. Eng. 1, 55-62 (2012)

11. L. Ming, J.X. Liu, X.F. Wang, Navig. China, 37, 4043 (2014)

12. Z.F. Li, Y. Sun, B. Wei, J, Dalian Marit. Univ. 44, 22-27 (2018) 
13. G. Cheng, Z.H. Xu, C.B. Tang, J, Guanzhou Marit. Inst. 25, 11-13 (2017)

14. Z.L. Qiu, C.X. Qi, G.P. Guo, Ship maneuvering (People's Communications Press, 1989)

15. L. Liang, P. Xun, C. Wan, International Conference on System Science, Engineering Design and Manufacturing Informatization, 114-117 (2011)
16. B.G. Hong, Ship Maneuvering (Dalian Maritime University Press, 2008)

17. F.T. Ren, X.M. Liu, J. Rong, et al., Traffic engineering (People's Communications Press, 2013)

18. Y.H. Dai, C.J. An, Z.G. Liao, et al., J. Transp. infor. saf. 28, 101-106(2010)

19. G.M. Ding, H.X. Liu, W.H. Zhao, et al., j. Chang'an Univ. 31, 77-80,85(2011) 